\title{
Prevalence and course of disease after lung resection in primary ciliary dyskinesia: a cohort \& nested case-control study
}

Panayiotis Kouis ${ }^{1}$, Myrofora Goutaki ${ }^{2}$, Florian S. Halbeisen ${ }^{2}$, Ifigeneia Gioti ${ }^{1}$, Nicos Middleton ${ }^{3}$, Israel Amirav ${ }^{4,25}$, on behalf of the Israeli PCD Consortium, Angelo Barbato ${ }^{5}$, on behalf of the Italian PCD Consortium, Laura Behan 6 , Mieke Boon ${ }^{7}$, Nagehan Emiralioglü, Eric G. Haarman", Bulent Karadag ${ }^{10}$, Cordula Koerner-Rettberg ${ }^{11}$, Romain Lazor ${ }^{12,13}$, on behalf of the Swiss PCD Group, Michael R. Loebinger ${ }^{14}$, Bernard Maitre ${ }^{15}$, on behalf of the French Reference Centre for Rare Lung Diseases, Henryk Mazurek ${ }^{16}$, Lucy Morgan ${ }^{17}$, Kim Gjerum Nielsen ${ }^{18}$, Heymut Omran ${ }^{19}$, Ugur Özçelik ${ }^{8}$, Mareike Price ${ }^{20}$, Andrzej Pogorzelski ${ }^{16}$, Deborah Snijders ${ }^{5}$, on behalf of the PCD Italian Consortium, Guillaume Thouvenin ${ }^{21}$, on behalf of the French Reference Centre for Rare Lung Diseases, Claudius Werner ${ }^{19,26}$, Zorica Zivkovic ${ }^{22,23}$, Claudia E. Kuehni ${ }^{2}$ and Panayiotis K. Yiallouros ${ }^{1,24^{*}}$

\begin{abstract}
Background: Lung resection is a controversial and understudied therapeutic modality in Primary Ciliary Dyskinesia (PCD). We assessed the prevalence of lung resection in PCD across countries and compared disease course in lobectomised and non-lobectomised patients.

Methods: In the international iPCD cohort, we identified lobectomised and non-lobectomised age and sexmatched PCD patients and compared their characteristics, lung function and BMI cross-sectionally and longitudinally.
\end{abstract}

Results: Among 2896 patients in the iPCD cohort, 163 from 20 centers (15 countries) underwent lung resection (5.6\%). Among adult patients, prevalence of lung resection was $8.9 \%$, demonstrating wide variation among countries. Compared to the rest of the iPCD cohort, lobectomised patients were more often females, older at diagnosis, and more often had situs solitus. In about half of the cases (45.6\%) lung resection was performed before presentation to specialized PCD centers for diagnostic work-up. Compared to controls $(n=197)$, lobectomised patients had lower FVC $z$-scores $(-2.41$ vs $-1.35, p=0.0001)$ and FEV1 $z$-scores $(-2.79$ vs $-1.99, p=0.003)$ at their first post-lung resection assessment. After surgery, lung function continued to decline at a faster rate in lobectomised patients compared to controls (FVC z-score slope: $-0.037 /$ year Vs $-0.009 /$ year, $p=0.047$ and FEV1 zscore slope: $-0.052 /$ year $V s-0.033 /$ year, $p=0.235$ ), although difference did not reach statistical significance for FEV1. Within cases, females and patients with multiple lobe resections had lower lung function.

Conclusions: Prevalence of lung resection in PCD varies widely between countries, is often performed before PCD diagnosis and overall is more frequent in patients with delayed diagnosis. After lung resection, compared to controls most lobectomised patients have poorer and continuing decline of lung function despite lung resection. Further studies benefiting from prospective data collection are needed to confirm these findings.

Keywords: Kartagener syndrome (MeSH), Ciliary motility disorders (MeSH), Lobectomy

\footnotetext{
*Correspondence: pyiall01@ucy.ac.cy

${ }^{1}$ Respiratory Physiology Laboratory, Medical School, University of Cyprus,

Nicosia, Cyprus

${ }^{24}$ Shakolas Educational Center of Clinical Medicine, Palaios Dromos

Lefkosias-Lemesou 215/6,2029 Aglantzia, Nicosia, Cyprus

Full list of author information is available at the end of the article
}

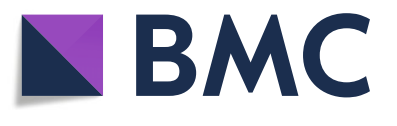

(c) The Author(s). 2019 Open Access This article is distributed under the terms of the Creative Commons Attribution 4.0 International License (http://creativecommons.org/licenses/by/4.0/), which permits unrestricted use, distribution, and reproduction in any medium, provided you give appropriate credit to the original author(s) and the source, provide a link to the Creative Commons license, and indicate if changes were made. The Creative Commons Public Domain Dedication waiver (http://creativecommons.org/publicdomain/zero/1.0/) applies to the data made available in this article, unless otherwise stated. 


\section{Background}

Primary Ciliary Dyskinesia (PCD) is a genetically heterogeneous disorder characterized by laterality defects and recurrent respiratory infections [1]. Bronchiectasis may develop already in childhood [2] and it is usually present in most adult PCD patients [3]. Late diagnosis is associated with worse clinical picture, [4-6] although even early diagnosis is followed by variable courses of lung function that are not linked to the level of lung function at diagnosis [7]. Management varies considerably between centers, as there are no evidence-based therapeutic guidelines for PCD [8]. Most of the current treatment protocols are extrapolated from Cystic Fibrosis (CF) studies.

Historically, in CF patients with localized bronchiectasis, lung resection has been proposed as a measure to decrease infection burden and limit the damage to the remaining lung [9-11]. However, a recent study from the US showed no improvement in lung function, hospital admissions or antibiotic use in CF patients after lung resection [12]. Similarly, a retrospective, small-scale study in children with non-CF bronchiectasis concluded that surgical treatment did not affect annual exacerbation rates and lung function but resulted in reductions the need for annual intravenous antibiotics [13]. Lung resection to treat PCD lung disease is generally not recommended, although it can be considered for selected cases of severe and localized bronchiectasis with recurrent suppurative infection, hemoptysis or specific infection [14]. Nevertheless, this approach remains controversial and to date, only two reports described the clinical course in PCD patients after lung resection, $[5,15]$ with conflicting results. The older study by Smit et al. compared 13 adult lobectomised PCD patients to 8 adult non-lobectomised PCD patients and did not find significant differences in respiratory symptoms between the two groups. Despite this, $85 \%$ of the lobectomised patients subjectively perceived the operation as beneficial [15]. A more recent study from Cyprus, compared 5 lobectomised PCD patients with 7 age-matched non-lobectomised PCD patients and reported that lobectomised patients had a more severe clinical picture and consistently lower lung function across time compared to non-lobectomised patients [5]. Both reports were single-center studies with small sample size and generalizability of their results is limited.

Ideally, the performance of a carefully conducted prospective study would be required to assess the impact of lung resection in PCD patients. However, due to the low prevalence of $\mathrm{PCD}$ and the rarity of lung resection, this approach is unrealistic. Alternatively, analysis of retrospective international registry data could offer important answers to this question. We used the international PCD cohort (iPCD) [16] to assimilate information from a large number of PCD patients across specialized centers in order to a) assess the prevalence of lung resection among
PCD patients, b) compare characteristics of lobectomised and non-lobectomised patients in the iPCD cohort and c) in a nested case-control study with more detailed data describe and identify predictors (sex, extent of lung resection, lung function prior lung resection) of disease course after lung resection.

\section{Methods \\ Population and study design}

We used a dataset from the iPCD cohort, a large retrospective international cohort study of $>3000$ PCD patients. All patients included in the iPCD cohort had diagnoses of other chronic lung diseases such as cystic fibrosis and primary immunodeficiency excluded. Additional details on iPCD cohort can be found elsewhere [16]. The cohort analysis included all patients whose data on lung resection status were available by May 2017. For each identified lobectomised patient, we randomly selected up to two controls, where available, stratified by age ( \pm 5 years), sex and center. In this manner, the groups of cases and controls were characterized by similar distributions over different levels of potential confounding variables such as age, sex and center (frequency matching design) [17]. Selection of cases and controls is presented in detail in Fig. 1. Information on available patient data and measurements as well as ethical approvals permitting the use of patient data is available in the online data supplement (Additional file 1).

\section{PCD diagnosis}

The iPCD Cohort includes data from patients diagnosed as early as 1964. Since then, availability of diagnostic tests and diagnostic criteria for PCD have evolved considerably. Originally, diagnosis was largely based on the presence of the Kartagener triad (bronchiectasis, sinusitis and situs inversus) and on transmission electron microscopy (TEM) findings. With time, High Speed Video Microscopy (HSVM), as well as nasal nitric oxide (nNO) and genetic analysis were introduced in the diagnostic work-up for PCD [18]. Nevertheless, even in recent years there is considerable variability in the availability of these tests between countries [8]. Towards better defining our study population, we classified all identified lobectomised patients and PCD controls in three diagnostic groups according to the recent guidelines of the ERS PCD Diagnostics Task Force [19]. More specifically, we classified PCD patients a) as "definite PCD" if they had hallmark EM findings and/or biallelic PCD genetic mutations, b) as "PCD highly likely" if they had abnormal HSVM findings and/or low nNO (using a cut-off of $77 \mathrm{nl} /$ min as suggested by Leigh MW et al., 2013), and c) as "clinical PCD" if they had a clinical phenotype suggestive of PCD but the PCD diagnostic algorithm was incomplete or diagnostic results were negative or ambiguous. All 


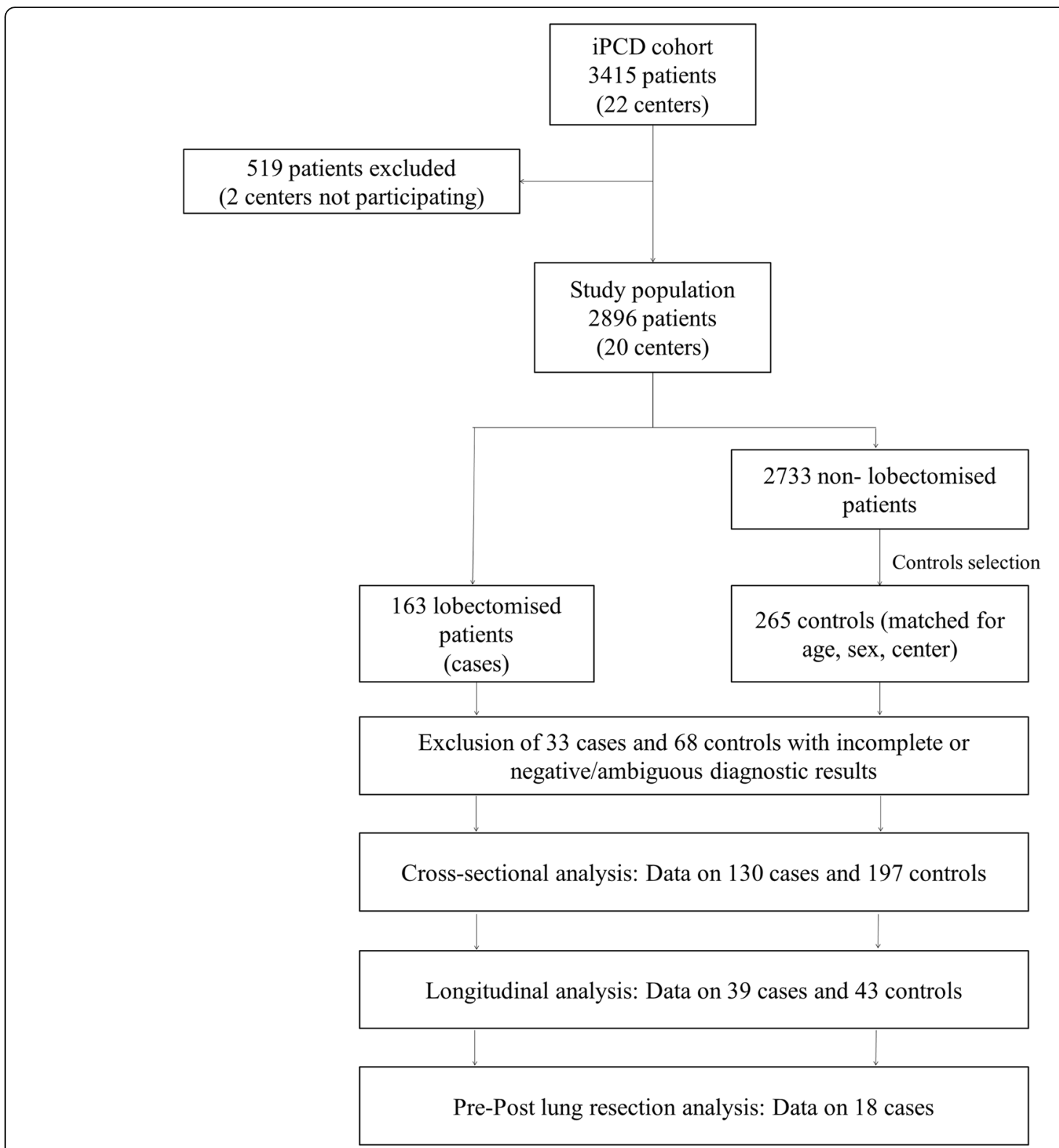

Fig. 1 Participants selection and data availability. Flowchart of participants' selection and data availability. From the 22 centers (3415 patients) that were included in the iPCD cohort, 20 centers (2896 patients) agreed to participate and provided data to the study. Of these 163 were patients that underwent lung resection. Of the remaining 2733 patients, we randomly selected 265 controls stratified by age ( \pm 5 years), sex and center. Data availability for cross-sectional and longitudinal case-control analysis as well as data availability for the within cases pre-post lung resection analysis is also displayed

patients classified as "clinical PCD" were excluded from statistical analysis and both the cross-sectional and longitudinal analysis is based on patients classified only as "definite PCD" or "PCD highly likely".

\section{Analysis}

\section{iPCD cohort study}

The prevalence of lung resection in PCD was calculated as percentage of all PCD patients in the dataset, as well as percentage of all PCD patients per study center.
Similarly, prevalence of lung resection among adults ( $\geq 18$ years) with PCD was calculated as percentage of total adult PCD patients in the dataset and per country. Basic characteristics of lobectomised patients were summarized as percentages or as medians and interquartile ranges (IQR), as appropriate. Differences in the distribution of characteristics between lobectomised and non-lobectomised patients in the iPCD cohort were investigated using chi-square test for categorical variables and Wilcoxon Sum Rank test for continuous variables. 
Nested case-control study - cross-sectional comparisons Diagnostic results and clinical characteristics of lobectomised patients (at first available post-lobectomy assessment) and non-lobectomised controls were compared using paired sample t-test and Wilcoxon Sum Rank test for normally and non-normally distributed variables respectively.

\section{Nested case-control study - longitudinal comparisons}

We explored disease course post-lung resection in lobectomised PCD patients compared to controls, using serial measurements of $\mathrm{FEV}_{1}, \mathrm{FVC}$ and BMI in a longitudinal mixed effects model. The model was defined by fixed effects for lung resection and age, an interaction term between lung resection and age and by random effects for intercepts and slopes (change per year). In addition, the frequency of positive sputum cultures for Pseudomonas aeruginosa was compared between cases and controls across different age groups (5-10, 10-15, $15-20,20-30$ and $30+$ ) by chi square test.

\section{Predictors of disease course within cases - longitudinal comparisons}

We also explored the effect of sex and number of lobes resected as potential predictors of adverse clinical course within the lobectomised group with two separate subgroup analyses. In the first subgroup analysis by sex, serial measurements of FEV1, FVC and BMI were analyzed with longitudinal mixed effect model defined as above and using a binary fixed effect term for sex while in the second subgroup analysis, the longitudinal mixed effect model used a binary fixed effect term for number of lobes resected.

\section{Pre- and post- lung resection comparisons}

In a small subgroup of lobectomised patients, we analyzed available lung function and BMI data before and after lung resection with the aim to provide insight to the critical question whether the adverse disease course of lobectomised patients is due to lung resection or to more severe disease phenotype prior lung resection. $\mathrm{FEV}_{1}, \mathrm{FVC}$ and BMI z-scores were plotted across time and the mean pre- and post- lung resection values were compared using paired samples t-test. Furthermore, mean lung function $\mathrm{z}$-scores of lobectomised patients at their last assessment prior to lung resection were compared with the mean of lung function measurements in controls obtained when they had the same age, using two-way analysis of variance to adjust for the effect of age. Lastly, the longitudinal mixed model analysis was repeated for this small group of patients and for an equal number of controls matched based on propensity score calculated using the variables for level of diagnostic certainty, sex, age at presentation and positive sputum culture. The calculation of the propensity score was carried out using the STATA "pscore" command [20].

Pairwise deletion was used to handle missing data in an analysis by analysis basis. Statistical comparisons were performed using STATA 12 (StataCorp, TX) and graphs were generated with Microsoft Excel.

\section{Results}

iPCD cohort study - prevalence of lung resection and patient characteristics

From 2896 PCD patients, followed-up in 20 centers across 15 countries, 163 (5.6\%) were reported to have undergone lung resection (Fig. 1). Among adults with PCD, lung resection was reported for 127 patients out of 1431 (8.9\%), whereas in the pediatric age-group only 36 out of $1465(2.5 \%)$ underwent surgical treatment. Wide variation was observed in lung resection prevalence among PCD centers (range: 0 to $17 \%$, Fig. 2, absolute numbers in Additional file 1: Table S1).

Lobectomised patients were significantly older at the time of the study (median age 24.9 vs 18.6 years, $p$ value $<0.001)$ and included more females $(55.4 \%$ vs $49.0 \%, p$-value $=0.150)$ than the non-lobectomised iPCD cohort (Table 1). In addition, lobectomised patients presented to the PCD specialist centers at an older age (11.5 vs 8.8 years, $p$-value $<0.001)$ and had less frequently laterality defects $(25 \%$ vs $44.1 \%, p$-value $<0.001)$ than the rest of the cohort. The number of lung resection procedures performed per decade in iPCD cohort patients seemed to be increasing ( $p$-value $<0.001)$ in the last decades (Table 1$)$. In most cases (83.5\%), lung resection was performed when the patients were in childhood and in about half of the cases (45.6\%) lung resection was performed before presentation to the specialized PCD centers for diagnostic work-up. Stratification by lung resections performed before or after presentation to a PCD center demonstrated that before 1990, almost all lung resections occurred prior presentation to a $\mathrm{PCD}$ center $(100 \%<1969$, 73\% during 1970-1979 and 89\% during 1980-1989). During the period 1990 to 2010, approximately $50 \%$ of lung resections occurred prior presentation to a PCD center, as opposed to only $18 \%$ after 2010 (Additional file 1: Table S3). In a quarter of the cases (22.1\%), lung resection was extended beyond one lobe, involving segments in 2 to 4 lobes, whereas the most frequently resected segments were at the right middle lobe (48.8\%) and left lower lobe (27.3\%) (Table 1).

\section{Nested case-control study - cross-sectional comparisons}

In total, 265 center, age and sex-matched non-lobectomised PCD patients were selected as controls from the 20 participating centers (Fig. 1). The targeted 1:2 casescontrols ratio was not achieved for every case due to lack of eligible controls in some centers. After exclusion 


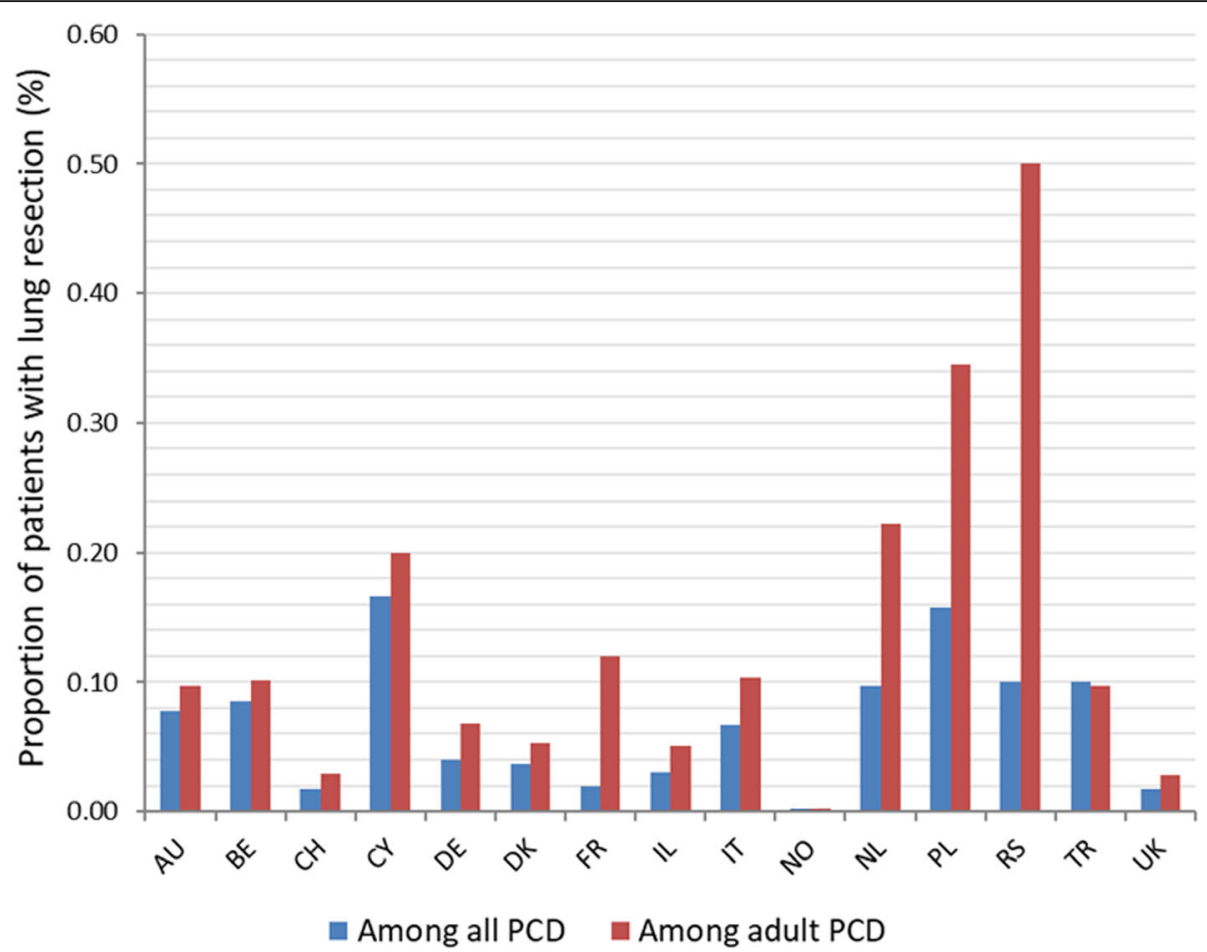

Fig. 2 The frequency of lung resection among patients in the iPCD cohort by participating centers. The prevalence of lung resection among PCD patients in the IPCD cohort across different centers. Prevalence among all PCD patients is denoted with dark color and prevalence among adult PCD patients is denoted with lighter pattern color. Absolute numbers are displayed in Additional file 1: Table S1 (Additional file 1). AU: Australia; BE: Belgium; CH: Switzerland; CY: Cyprus; DE1: Bochum, Germany, DE2: Muenster, Germany; DE3: Hannover, Germany; DK: Denmark; FR: France; IL: Israel; IT: Italy; NL: the Netherlands; NO: Norway; PL: Poland; RS: Serbia; TR1: Istanbul, Turkey; TR2: Ankara, Turkey; UK1: Paediatric Pulmonology Dept, Brompton, UK; UK2: Adult Pulmonology Dept, Brompton, UK; UK3: Southampton, UK

of patients characterized by ambiguous or incomplete diagnostic results ("clinical PCD"), a total of 130 lobectomised and 197 controls were compared. Although nasal nitric oxide was low in both cases and controls, it was somewhat higher in lobectomised patients (median: 16 vs $10 \mathrm{nl} / \mathrm{min}, p$-value $=0.013$ ). Ciliary ultrastructure and motility were not different between the two groups (Additional file 1: Table S2). Crosssectional comparisons of clinical characteristics of controls at presentation with those of lobectomised patients at their first available post-lobectomy assessment, revealed higher prevalence of bronchiectasis $(95.7 \%$ vs $76.1 \%, p$-value $=0.004)$ and chronic cough $(96.8 \%$ vs $88.4 \%, p$-value $=0.023)$ but less wheezing $(39.7 \%$ vs $59.2 \%, p$-value $=0.016)$ in the lobectomised cohort (Table 2). Compared to controls, lobectomised patients had lower FVC $(-2.41$ vs $-1.35, p=0.0001)$ and $\mathrm{FEV}_{1}(-2.79$ vs $-1.99, p=0.003) \mathrm{z}$-scores at baseline (first post-lobectomy assessment) but no difference in BMI zscores $(0.03$ vs $-0.09, p$-value $=0.599)$.

\section{Nested case-control study - longitudinal comparisons}

In a longitudinal mixed model analysis, we included 39 lobectomised PCD patients and 43 non-lobectomised controls who had available two or more repeated measurements of FVC, $\mathrm{FEV}_{1}$ and BMI. The mean time interval with available longitudinal measurements for each lobectomised patient was 9.20 years (95\% CI: 7.40-11.01) while the mean interval for each control patient was 10.82 years $(95 \% \mathrm{CI}$ : 8.22-13.41). The difference between the two values was not statistically significant ( $p$-value: 0.310$)$. We found no difference at the level (intercept) of FVC $(-1.00$ vs $-0.75, p$ value $=0.489)$ and $\operatorname{FEV}_{1}(-1.47$ vs $-1.12, p$-value $=0.346)$ between lobectomised and control patients (Table 3). However, both control and lobectomised groups, displayed loss of lung function with time. Although lung function decline in the lobectomised patients after lung resection was somewhat steeper in terms of FVC (z-score slope: - 0.037/ year vs $-0.009 /$ year, $p=0.047$ ) and $\mathrm{FEV}_{1}$ (z-score slope: $0.052 /$ year vs -0.033 /year, $p=0.235$ ), the difference in the latter did not reach statistical significance. In contrast, BMI remained stable post-lung resection in lobectomised patients compared to controls (z-score slope: 0.024/year vs $0.015 /$ year, $p=0.507$ ) (Table 3). Lobectomised patients had also more frequent $(77 / 263,29.3 \%)$ positive sputum cultures for Pseudomonas aeruginosa compared to controls (66/487, $13.6 \%)$ ( $p$-value $<0.001)$ that was evident in age-groups after the age of 10 years (Additional file 2: Figure S1). 
Table 1 Features of lobectomised and non-lobectomised patients in the iPCD cohort

\begin{tabular}{|c|c|c|c|c|}
\hline Variable & $\begin{array}{l}\text { Lobectomised PCD } \\
(n=130) \text { Median (IQR) }\end{array}$ & & $\begin{array}{l}\text { iPCD Cohort }(n=2733)^{\mathrm{a}} \\
\text { Median (IQR) }\end{array}$ & $p$-value \\
\hline Age at Presentation $(n=118)$ & $11.5(6.5,20.5)$ & & $8.8(4.0,15.8)$ & $<0.001 \dagger$ \\
\hline Current Age $(n=130)$ & $24.9(18.1,40.1)$ & & $18.6(12.1,28.3)$ & $<0.001+$ \\
\hline$\%$ Female & $72 / 130(55.4 \%)$ & & $1302 / 2661(48.9 \%)$ & $0.150 \neq$ \\
\hline Situs Inversus & $27 / 108(25.0 \%)$ & & 787/1783 (44.1\%) & $<0.001 \neq$ \\
\hline Age at lung resection $(n=121)$ & $11.9(7.7,16.0)$ & & & \\
\hline Lung resection prior to presentation & $52 / 114(45.6 \%)$ & & & \\
\hline Lung resection in childhood & $101 / 121(83.5 \%)$ & & & \\
\hline \multirow{6}{*}{$\begin{array}{l}\text { Frequency of lung resection performance } \\
\text { per decade }(n=121)\end{array}$} & $<1969$ & 4 & & $<0.001 \neq$ \\
\hline & 1970-1979 & 11 & & \\
\hline & 1980-1989 & 9 & & \\
\hline & 1990-1999 & 24 & & \\
\hline & $2000-2009$ & 34 & & \\
\hline & 2010-2017 & 39 & & \\
\hline \multirow[t]{8}{*}{ Extent of lung resection $(n=95)$} & One Lobe: 74/95 & & & \\
\hline & $(77.9 \%)$ & & & \\
\hline & Two lobes: $17 / 95$ & & & \\
\hline & $(17.9 \%)$ & & & \\
\hline & Three Lobes: 2/95 & & & \\
\hline & $(2.1 \%)$ & & & \\
\hline & Four Lobes: 2/95 & & & \\
\hline & $(2.1 \%)$ & & & \\
\hline \multirow[t]{6}{*}{ Site of lung resection ${ }^{\mathrm{b}}(n=95)$} & RML: 59/121 (48.8\%) & & & \\
\hline & LLL: 33/121 (27.3\%) & & & \\
\hline & RLL: $13 / 121$ (10.7\%) & & & \\
\hline & Lingula: 9/121 (7.4\%) & & & \\
\hline & RUL: 5/121 (4.1\%) & & & \\
\hline & LUL: 2/121 (1.7\%) & & & \\
\hline
\end{tabular}

Denominators indicate number of subjects with available data on the specific parameter

RUL: Right Upper Lobe, RML: Right Middle Lobe, RLL: Right Lower Lobe, LUL: Left Upper Lobe, LLL: Left Lower Lobe

${ }^{a}$ Full iPCD cohort that participated in the study, excluding lobectomised patients (May 2017)

${ }^{b}$ Denominator reflects the total number of resected lobes. Some patients had more than one lobe resected. For one patient (one with one lobe resected) the

exact site was not reported

†Wilcoxon Sum Rank Test

‡Pearson Chi Square Test

\section{Predictors of disease course}

Within lobectomised patients, subgroup analysis by sex indicated sharp differences between males and females in the level (intercept) of $\mathrm{FEV}_{1}(-0.94 \mathrm{vs}-1.96, p$-value $=$ $0.064)$ and FVC $(-0.68$ vs $-1.65, p$-value $=0.125) \mathrm{z}$-scores after lung resection, with females having significantly worse lung function. Patients of both sexes continued to lose lung function with time after lung resection but changes were somewhat steeper in males, with significantly worse slope for $\mathrm{FEV}_{1}(-0.08 /$ year vs -0.03 / year, $p$-value $=0.026)$ but not for FVC $(-0.05 /$ year vs $0.021 /$ year, $p$-value $=0.263$ ) (Table 4$)$. In subgroup analysis by the number of lobes resected, we found unfavorable differences at the level of FVC $(-0.75$ vs $-2.49, p$-value $=$ $0.040)$ and $\mathrm{FEV}_{1}(-1.10 \mathrm{vs}-2.89, p$-value $=0.012)$ z-scores intercepts in patients who underwent multiple lobes resection in comparison to patients who had only one lobe resected. However, subsequent lung function decline with ageing was not different between the two groups (Table 4).

In a subgroup of 18 lobectomised patients, lung function and BMI data before and after lung resection were available. The basic characteristics of these 18 patients are presented in Additional file 1: Table S4. The individual FVC, $\mathrm{FEV}_{1}$ and BMI $\mathrm{z}$-scores trends as well as the average trend for these patients across time, before and after lung resection appear in Fig. 3 in comparison to 
Table 2 Cross-sectional characteristics of lobectomised PCD patients (on first post-lung resection assessment) compared to matched controls

\begin{tabular}{llll}
\hline & Lobectomised PCD $(n=130)$ & Controls PCD $(n=197)$ & $p$-valuet \\
\hline FVC Z score & $-2.41(-2.91,-1.90)$ & $-1.35(-1.70,-1.00)$ & $0.0001 \neq$ \\
FEV1 Z score & $-2.79(-3.25,-2.32)$ & $-1.99(-2.32,-1.65)$ & $0.003 \neq$ \\
BMI Z score & $0.03(-0.34,0.40)$ & $-0.09(-0.38,0.19)$ & $0.599 \neq$ \\
Sputum Culture & & & \\
$\quad$ Any Pathogen & $60 / 73(82.2 \%)$ & $74 / 92(80.4 \%)$ & 0.774 \\
$\quad$ Pseudomonas & $20 / 73(27.4 \%)$ & $15 / 92(16.3 \%)$ & 0.083 \\
Bronchiectasis & $44 / 46(95.7 \%)$ & $86 / 113(76.1 \%)$ & 0.004 \\
Congenital Heart Disease & $10 / 86(11.6 \%)$ & $13 / 126(10.3 \%)$ & 0.763 \\
NRDS & $37 / 78(47.4 \%)$ & $51 / 114(44.7 \%)$ & 0.712 \\
Chronic Cough & $92 / 95(96.8 \%)$ & $99 / 112(88.4 \%)$ & 0.023 \\
Sputum & $78 / 82(95.1 \%)$ & $73 / 81(90.1 \%)$ & 0.222 \\
Wheezing & $25 / 63(39.7 \%)$ & $58 / 98(59.2 \%)$ & 0.016 \\
Pneumonia & $11 / 66(16.7 \%)$ & $35 / 120(29.2 \%)$ & 0.059 \\
Hemoptysis & $2 / 58(3.5 \%)$ & $0 / 67(0 \%)$ & 0.125 \\
Rhinorrhea & $68 / 79(86.1 \%)$ & $111 / 123(90.2 \%)$ & 0.363 \\
\hline
\end{tabular}

Denominators indicate number of subjects with available data on the specific parameter NRDS: Neonatal Respiratory Distress Syndrome +Pearson Chi Square Test, with the exception of FVC, FEV1 and BMI fPaired Samples T test

the respective trends of propensity score matched controls. Average lung function $\mathrm{z}$-scores before lung resection were significantly higher when compared to $\mathrm{z}$ scores after lung resection $\left(\mathrm{FEV}_{1}:-1.77 \mathrm{Vs}-2.69 p\right.$ value $<0.001$, FVC: -1.16 Vs $-1.99, p$-value $=0.003$ ), whereas BMI z-scores pre and post-lung resection did not differ $(0.33$ Vs $0.22, p$-value $=0.669)$. Mean lung function z-scores of lobectomised patients at their last assessment prior lung resection did not differ from the mean lung function of matched non-lobectomised patients obtained when they had the same age (mean difference in $\mathrm{FEV}_{1}: 0.00075, p$-value $=0.820$, mean difference in FVC:
$0.34, p$-value $=0.512$ ). The analysis of FVC, FEV1 and BMI z-score decline between the 18 lobectomised patients and their respective propensity score matched controls demonstrated a steeper decline per year in the lobectomised patients especially in FVC z-scores $(-0.080$ Vs $0.010, p$ value $=0.006)$ and not so in FEV1 z-scores $(-0.099 \mathrm{Vs}-$ $0.029, p$-value $=0.071)($ Additional file 1: Table S5).

\section{Discussion}

In this study, we present the first cumulative retrospective data on lung resection in a large number $(n=163)$ of PCD patients, reported from 20 centers across 15 countries.

Table 3 Change in lung function and BMl over time (post-lung resection) in lobectomised PCD patients $(n=39)$ and controls $(n=43)$

\begin{tabular}{|c|c|c|c|c|c|}
\hline Outcome & Group & Intercept (95\% Cl) & $p$-value & Change per year $(95 \% \mathrm{Cl})$ & $p$-value* \\
\hline \multirow[t]{4}{*}{ FVC Z score } & Lobectomised & -1.00 & 0.489 & -0.037 & 0.047 \\
\hline & & $(-2.17,-0.17)$ & & $(-0.09,0.01)$ & \\
\hline & Controls & -0.75 & & -0.009 & \\
\hline & & $(-1.20,-0.29)$ & & $(-0.03,0.01)$ & \\
\hline \multirow[t]{4}{*}{ FEV1 Z score } & Lobectomised & -1.47 & 0.346 & -0.052 & 0.235 \\
\hline & & $(-2.65,-0.28)$ & & $(-0.10,0.00)$ & \\
\hline & Controls & -1.12 & & -0.033 & \\
\hline & & $(-1.58,-0.66)$ & & $(-0.05,-0.02)$ & \\
\hline \multirow[t]{4}{*}{ BMI Z score } & Lobectomised & -0.44 & 0.790 & 0.024 & 0.507 \\
\hline & & $(-1.49,0.61)$ & & $(-0.02,0.07)$ & \\
\hline & Controls & -0.35 & & 0.015 & \\
\hline & & $(-0.74,0.04)$ & & $(-0.002,0.03)$ & \\
\hline
\end{tabular}

* $P$ value for interaction, testing whether the relationship between independent variables (FVC, $\mathrm{FEV}$, BMI) and time is different between Lobectomised and Controls 
Table 4 Subgroup Analysis: Change in lung function and BMl over time (post-lung resection) by sex ( $n=39$ ) and by extent of lung resection $(n=35)$

\begin{tabular}{|c|c|c|c|c|c|}
\hline Outcome & Subgroup & Intercept $(95 \% \mathrm{Cl})$ & $p$-value & $\begin{array}{l}\text { Change per year } \\
(95 \% \text { Cl) }\end{array}$ & $p$-value* \\
\hline \multirow[t]{4}{*}{ FVC Z score } & Males & -0.68 & 0.125 & -0.049 & 0.263 \\
\hline & & $(-1.57,0.22)$ & & $(-0.09,-0.01)$ & \\
\hline & Females & -1.65 & & -0.021 & \\
\hline & & $(-3.79,0.49)$ & & $(-0.11,0.07)$ & \\
\hline \multirow[t]{4}{*}{$\mathrm{FEV}_{1}$ Z score } & Males & -0.94 & 0.064 & -0.08 & 0.026 \\
\hline & & $(-1.71,-0.17)$ & & $(-0.12,-0.05)$ & \\
\hline & Females & -1.96 & & -0.03 & \\
\hline & & $(-3.81,-0.11)$ & & $(-0.11,0.04)$ & \\
\hline \multirow[t]{4}{*}{ BMI Z score } & Males & -0.04 & 0.180 & 0.02 & 0.967 \\
\hline & & $(-0.71,0.62)$ & & $(-0.01,0.05)$ & \\
\hline & Females & -0.68 & & 0.02 & \\
\hline & & $(-2.27,-0.92)$ & & $(-0.05,0.09)$ & \\
\hline \multirow[t]{4}{*}{ FVC Z score } & Single Lobe & -0.75 & 0.040 & -0.03 & 0.645 \\
\hline & & $(-1.38,-0.13)$ & & $(-0.06,-0.01)$ & \\
\hline & Multiple Lobes & -2.49 & & -0.05 & \\
\hline & & $(-4.79,-0.21)$ & & $(-0.14,0.04)$ & \\
\hline \multirow[t]{4}{*}{ FEV1 Z score } & Single Lobe & -1.10 & 0.012 & -0.06 & 0.955 \\
\hline & & $(-1.61,-0.60)$ & & $(-0.08,-0.03)$ & \\
\hline & Multiple Lobes & -2.89 & & -0.06 & \\
\hline & & $(-4.78,-0.99)$ & & $(-0.14,0.02)$ & \\
\hline \multirow[t]{4}{*}{ BMI Z score } & Single Lobe & -0.15 & 0.456 & 0.02 & 0.042 \\
\hline & & $(-0.64,0.35)$ & & $(0.01,0.04)$ & \\
\hline & Multiple Lobes & -0.62 & & -0.02 & \\
\hline & & $(-2.35,1.12)$ & & $(-0.08,0.04)$ & \\
\hline
\end{tabular}

* $P$ value for interaction, testing whether the relationship between independent variables ( $\left.F V C, F_{1}, B M I\right)$ and time is different within the lobectomised patients between males and females (Subgroup Analysis 1) and between single and multiple lobes resected (Subgroup Analysis 2)

Despite the anecdote that lobectomy is uncommon in PCD, in this representative sample of 2896 PCD patients, we report an overall prevalence of lobectomy nearly $6 \%$, whereas in adult PCD populations from one fifth of the centers prevalence exceeds $20 \%$. Prevalence rates of lung resection in single-center reports, $[9,10,12]$ in CF cohorts are much lower (3\%) compared to our series. There are no large scale, international data either on the prevalence or on long-term outcomes of lung resection in CF.

The characteristics of lobectomised patients provide possible explanations for the high prevalence of lung resection in PCD. Lobectomised PCD patients were diagnosed at an older age and had much less frequently laterality defects, indicating the difficulty to establish the diagnosis in these patients, in comparison to the rest of the iPCD cohort. In fact, in about half of the cases, lung resection was performed prior diagnosis, which suggests that in these patients the decision of lung resection was taken at a time when the nature of chronic lung disease was probably unknown. Interestingly, lung resections prior to diagnosis were performed more frequently during the earlier decades with available data (before 1990), in a period where awareness and knowledge about PCD was scarce [4]. This seems to have changed in later decades (after 1990), where the opposite trend was observed with gradually less lung resections performed prior to presentation. After 2010, it appears that very few PCD patients underwent lung resection prior to presentation (18\%), although the latter figure may be an underestimation of the reality as some undiagnosed PCD patients undergoing lung resection in the last few years might have not been diagnosed by PCD centers yet. Differences in age of diagnosis as well as in surgical and medical care of bronchiectasis across countries may explain the differences in the rates of lung resection across countries. Persistent atelectasis and consolidation, especially at the middle and lower lobes, are common features in PCD [21, 22]. In comparison to CF, MRI and 


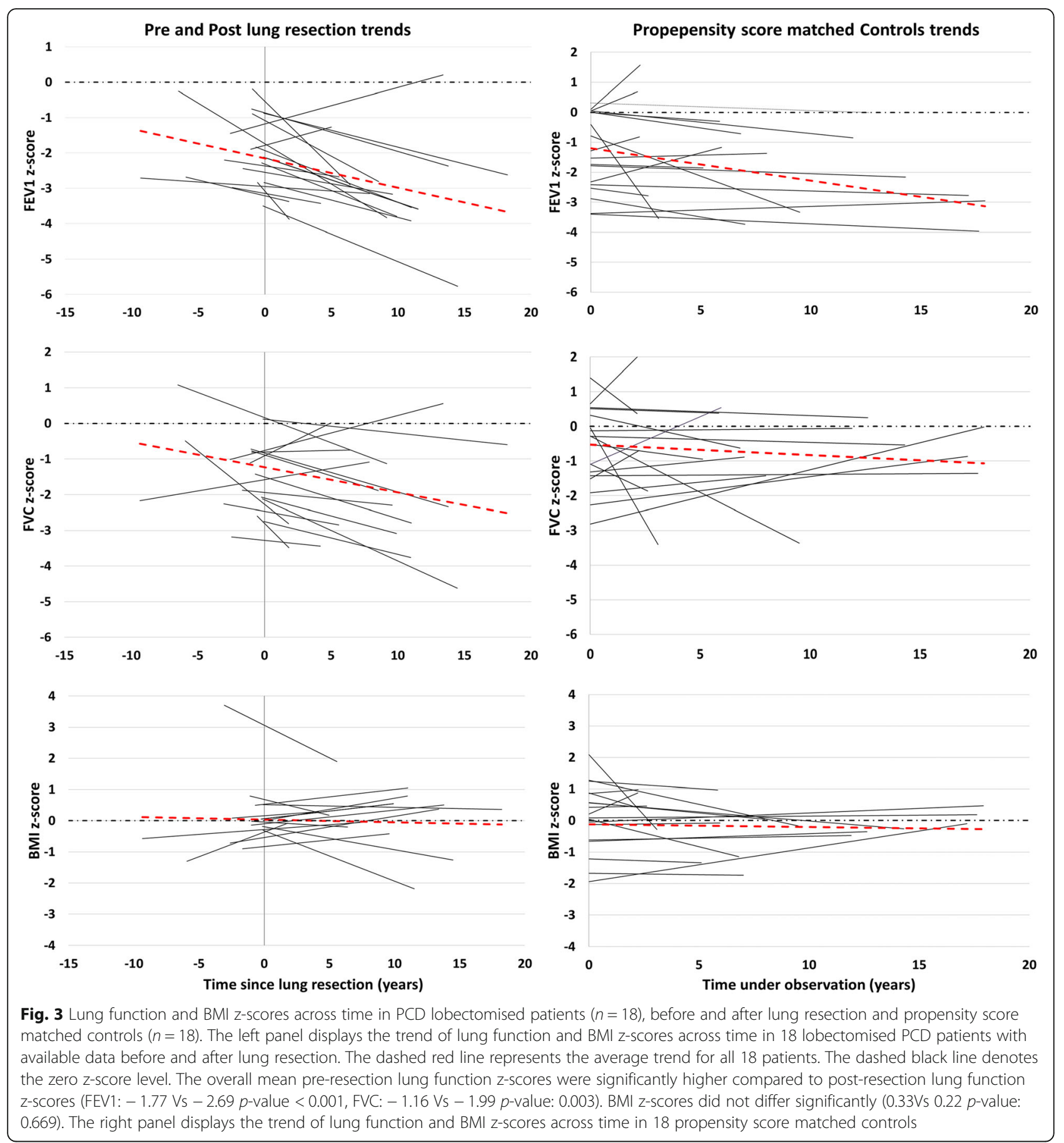

CT scores for lung collapse/consolidation in PCD are of higher severity, [23-25] which may be a contributing factor to the more frequent performance of lung resection in PCD. RML was by far the most frequently resected lobe, indicating the frequency and severity of the involvement of this lobe in PCD lung disease. Previous reports found associations between bronchiectasis development in RML and unfavorable clinical outcomes [26, 27].
Our study also provides important insight in PCD disease course after lung resection. Lobectomised patients have higher prevalence of bronchiectasis and lower FVC and FEV1 at first post-lung resection assessment when compared to their matched controls, as may be expected after loss of lung tissue. Despite performing lung resection to treat chronic lung disease complications, most lobectomised patients continue to display loss of lung function with time after surgery, which is somewhat 
steeper in terms of FVC but not FEV1, in comparison to non-lobectomised controls. Knowledge of this may have important implications at the stage of considering the decision to undertake such an intervention. This trend was also evident when we compared the course of lung function between the 18 patients that had available lung function data pre and post lung resection and 18 propensity score matched controls. Lobectomised patients also display more frequent growth of Pseudomonas aeruginosa in their sputa, which was shown in one adult PCD study [28] to be a poor predictor of lung function, although this was not confirmed in other studies [29]. Although a previous iPCD study [30] showed association of lung function with BMI, we found that BMI had a stable course in our lobectomised and control groups.

Patients who underwent multiple lobes resection had much lower level of lung function in comparison to those with only one lobe resected. This is not surprising given that more lung tissue was removed, but interestingly these patients display similar loss of lung function after lung resection with patients who underwent single lobe resection. Being female has been recently acknowledged as a predictor for poor lung function in adult PCD patients, [28] and it is a well-known predictor of adverse disease course in CF. [31, 32] In this series, being female is associated with adverse post-lung resection lung function, although loss of lung function in subsequent years is somewhat more profound in males who seem to lose more rapidly thereafter the excess lung function they have in comparison to females.

In a small subgroup of 18 patients, we had some indications that the mean lung function prior lung resection declined after surgery. However, lung volume reduction by the operation is confounding the value of lung function as a reliable parameter for the comparison of disease course pre and post operation. Pre-resection lung function was similar to the lung function of matched non-lobectomised PCD patients, suggesting that at least in terms of lung function the disease course before surgery was similar to non-lobectomised patients. Unfortunately, we have no detailed data on other important clinical indices prior lung resection, such as extent of bronchiectasis and clinical morbidity that are also key parameters of clinical severity. Interestingly, in contrast to the adverse lung function trend in the whole group, evaluation of individual $\mathrm{FVC}$ and $\mathrm{FEV}_{1}$ trends before and after lung resection in a minority of patients demonstrated improved lung function after surgery. This finding suggests that surgical intervention may be a worthwhile option in carefully selected PCD patients with severe localized symptomatic bronchiectasis. More studies are needed to elicit predisposing factors for the favorable post-lung resection course in this minority of patients.
The major strength of this study is the provision of data on a large, representative, international sample of PCD patients who underwent surgery to treat bronchiectasis, demonstrating continuing lung function decline after surgery. However, the study was retrospective and although effort was made to collect all relevant data based on a predefined protocol, not only from the iPCD registry but also by contacting the local principal investigators at the collaborating centers, we cannot exclude that some data were missed. In addition, given its retrospective nature, the study did not benefit from a standardized diagnostic protocol (not all PCD patients underwent the same tests across different centers) or standardized lung function testing procedures as described by the ERS/ATS recommendations [33]. The multicenter nature of the study also might introduce centerrelated heterogeneity in the collected parameters that we tried to control by recruiting for each lobectomised patient two controls matched for age, sex and center, although this was not possible for all cases. Furthermore, participating patients had different stages of disease and underwent different kinds of surgical intervention, introducing variabilities that may affect the outcomes, which are impossible to assess and control in a retrospective study. Overall, although this is the first study that examines and provides insights into the pragmatic outcomes of lung resection in a large number of PCD patients across many international centers, the retrospective nature of the data as well as the small sample size available for some of the subgroup analyses warrant caution in the generalization of the results.

\section{Conclusions}

Prevalence of lung resection for treating bronchiectasis in PCD varies widely between countries and is unexpectedly high in some cohorts of adult patients. Historically, lung segments were removed from patients who were subsequently diagnosed with PCD. These patients tended to not have other clues for the diagnosis such as situs inversus. PCD diagnosis should be considered in all patients with bronchiectasis, especially those with bronchiectasis severe enough for lobectomy to be considered. This study demonstrates that lung resection in PCD, especially in female patients, is associated with more severe impairment of lung function, which continues to decline after lung resection. Further studies, benefiting from prospective data collection are needed to confirm these findings.

\section{Supplementary information}

Supplementary information accompanies this paper at https://doi.org/10. 1186/s12931-019-1183-y

Additional file 1. Online Data Supplement (Text and Tables).

Additional file 2: Figure S1. Frequency of Pseudomonas positive cultures in PCD lobectomised patients $(n=24)$ and controls $(n=43)$. Frequency of Pseudomonas positive cultures among lobectomised 
patients (displayed in black) and among controls (displayed in grey). The proportion of positive sputum cultures for Pseudomonas aeruginosa in each group was calculated as the sum of individual patients' proportions of positive cultures weighted by the ratio of the number of cultures taken from the individual patient versus the total number of cultures in the specific age group.

\section{Acknowledgments}

We are grateful to all patients in the PCD cohort and their families and especially to the PCD patient organisations for their continuing support. We would also like to thank all the researchers that were involved in data collection and data entry during the development of the iPCD cohort dataset

\section{Authors' contributions}

PKY conceived the study hypothesis and PKY, CK, PK and MG developed the study concept. PK, MG, FSH and IG cleaned and standardized the data. PK and FSH performed the statistical analysis under the guidance of NM. All other authors participated in discussions for the development of the study and contributed data. PK, PKY, MG, FSH, CK prepared the first draft of the manuscript and all other authors contributed towards the interpretation of the findings and critically revised the manuscript. All authors read and approved the final manuscript.

\section{Funding}

This study was supported by EU 7th Framework Program EC-GA No. 305404 BESTCILIA and COST action BEAT-PCD (BM1407). The primary ciliary dyskinesia (PCD) research at the Institute of Social and Preventive Medicine (ISPM) in Bern is supported by the Swiss National Science Foundation (SNF 320030_173044). This work was supported by Ministry of Science Republic of Serbia, grant No III 41004. In France, this work was supported by the Institut National de la Sante et de la Racherche Medicale (INSERM), the RaDiCO funded by the French National Research Agency under the specific program "Investments for the future" (Cohort grant agreement ANR-10-COHO-0003). The sponsors had no role in the design of the study, the collection and analysis of the data, or the preparation of the manuscript.

\section{Availability of data and materials}

The datasets used and analyzed during the current study are available from the corresponding author on reasonable request.

\section{Ethics approval and consent to participate}

Local Primary Investigators (PIs) received ethics approval and informed consent for collecting patient data for local research use or national registries. In countries where informed consent is required even for anonymised observational data, Pls are responsible for obtaining ethics approval and informed consent in their country for the contribution of their anonymised data to the iPCD Cohort for research purposes. The iPCD cohort database is maintained at University of Bern (Approval Number: 060/15, Kantonale Ethikkommission für die Forschung Bern).

\section{Consent for publication}

Not applicable.

\section{Competing interests}

DS and MB report grants from Italian Health Ministry and Horizon 2020 respectively, outside the submitted work. All other authors wish to declare that they have no competing interests.

\section{Author details}

${ }^{1}$ Respiratory Physiology Laboratory, Medical School, University of Cyprus, Nicosia, Cyprus. ${ }^{2}$ Institute of Social and Preventive Medicine, University of Bern, Bern, Switzerland. ${ }^{3}$ Department of Nursing, School of Health Sciences, Cyprus University of Technology, Limassol, Cyprus. ${ }^{4}$ Department of Pediatrics University of Alberta Edmonton, Edmonton, Canada. ${ }^{5}$ Primary Ciliary Dyskinesia Centre, Department of Women's and Children's Health (SDB), University of Padova, Padova, Italy. ${ }^{6}$ Primary Ciliary Dyskinesia Centre, University Hospital Southampton, NHS Foundation Trust and University of Southampton, Southampton, UK. ${ }^{7}$ Department of Paediatrics \& Paediatric Pulmonology, University Hospital Gasthuisberg Leuven, Leuven, Belgium.
${ }^{8}$ Pediatric Pulmonology, Hacettepe University, Ankara, Turkey. ${ }^{9}$ Department of pediatric pulmonology, Emma Children's Hospital, Amsterdam UMC, Vrije Universiteit, Amsterdam, The Netherlands. ${ }^{10}$ Department of Pediatric Pulmonology, Marmara University, School of Medicine, Istanbul, Turkey. ${ }^{11}$ Department of Paediatric Pulmonology, University Children's Hospital of Ruhr University Bochum, Bochum, Germany. ${ }^{12}$ Department of Respiratory Medicine, Lausanne University Hospital, Lausanne, Switzerland. ${ }^{13}$ Department of Respiratory Medicine, National Reference Centre for Rare Pulmonary Diseases, Lyon, France. ${ }^{14}$ Host Defence Unit, Royal Brompton and Harefield NHS Foundation Trust, London, UK. ${ }^{15}$ Hopital intercommunal de Créteil, Service de Pneumologie, DHU ATVB, Université Paris Est Créteil, Paris, France. ${ }^{16}$ Klinika Pneumonologii i Mukowiscydozy, Instytut Gruźlicy i ChoróbPłuc, Rabka, Poland. ${ }^{17}$ Department of Respiratory Medicine, Concord Hospital Clinical School, University of Sydney, Sydney, Australia. ${ }^{18}$ Danish PCD Centre Copenhagen, Paediatric Pulmonary Service, Copenhagen University Hospital, Copenhagen, Denmark. ${ }^{19}$ Department of General Paediatrics and Adolescent Medicine, University Hospital Muenster, Muenster, Germany. ${ }^{20} \mathrm{Clinic}$ for Paediatric pulmonology, Allergiology and Neonatology, Hannover Medical School, Hannover, Germany. ${ }^{21}$ Service de pneumologie pédiatrique, Hôpital Trousseau, APHP, Sorbonne Université, INSERM, Centre de Recherche Saint-Antoine, CRSA, Paris, France. ${ }^{22}$ Children's Hospital for Lung Diseases and TB, Medical Centre "Dr Dragisa Misovic", Belgrade, Serbia. ${ }^{23}$ Faculty of Pharmacy Novi Sad, Business Academy in Novi Sad, Novi Sad, Serbia. ${ }^{24}$ Shakolas Educational Center of Clinical Medicine, Palaios Dromos Lefkosias-Lemesou 215/6,2029 Aglantzia, Nicosia, Cyprus. ${ }^{25}$ Dana-Dwek Children's Hospital, Tel Aviv Medical Center, Tel Aviv, Israel. ${ }^{26}$ Department of Pediatrics, Helios Hospital Schwerin, Schwerin, Germany.

Received: 25 March 2019 Accepted: 10 September 2019

Published online: 18 September 2019

\section{References}

1. Knowles MR, Daniels LA, Davis SD, Zariwala MA, Leigh MW. Primary ciliary dyskinesia. Recent advances in diagnostics, genetics, and characterization of clinical disease. Am J Respir Crit Care Med. 2013;188(8):913-22.

2. Magnin ML, Cros P, Beydon N, Mahloul M, Tamalet A, Escudier E, Clément A, Pointe L, Ducou H, Blanchon S. Longitudinal lung function and structural changes in children with primary ciliary dyskinesia. Pediatr Pulmonol. 2012; 47(8):816-25.

3. Honoré I, Burgel P. Primary ciliary dyskinesia in adults. Rev Mal Respir. 2016; 33(2):165-89.

4. Coren M, Meeks M, Morrison I, Buchdahl R, Bush A. Primary ciliary dyskinesia: age at diagnosis and symptom history. Acta Paediatr. 2002;91(6):667-9.

5. Yiallouros PK, Kouis P, Middleton N, Nearchou M, Adamidi T, Georgiou A, Eleftheriou A, loannou P, Hadjisavvas A, Kyriacou K. Clinical features of primary ciliary dyskinesia in Cyprus with emphasis on lobectomized patients. Respir Med. 2015;109(3):347-56.

6. Ellerman A, Bisgaard H. Longitudinal study of lung function in a cohort of primary ciliary dyskinesia. Eur Respir J. 1997;10(10):2376-9.

7. Marthin JK, Petersen N, Skovgaard LT, Nielsen KG. Lung function in patients with primary ciliary dyskinesia: a cross-sectional and 3-decade longitudinal study. Am J Respir Crit Care Med. 2010:181(11):1262-8.

8. Strippoli MP, Frischer T, Barbato A, Snijders D, Maurer E, Lucas JS, Eber E, Karadag B, Pohunek P, Zivkovic Z, Escribano A, O'Callaghan C, Bush A, Kuehni CE. ERS task force onPrimary ciliary dyskinesia in children: management of primary ciliary dyskinesia in European children: recommendations and clinical practice. Eur Respir J. 2012;39(6):1482-91.

9. Camargos P, Le Bourgeois M, Revillon Y, Tatsuo E, Sermet-Gaudelus I, Scheinmann $P$, de Blic J. Lung resection in cystic fibrosis: a survival analysis. Pediatr Pulmonol. 2008;43(1):72-6.

10. Lucas J, Connett GJ, Lea R, Rolles CJ, Warner JO. Lung resection in cystic fibrosis patients with localised pulmonary disease. Arch Dis Child. 1996;74(5):449-51.

11. Lucas JS, Connett GJ, Fairhurst J. Long term results of lung resection in cystic fibrosis patients with localised lung disease. Arch Dis Child. 2002;86(1):66.

12. Sheikh SI, McCoy K, Ryan-Wenger NA, Patel A, Kirkby S. lobectomy in patients with cystic fibrosis. Can Respir J. 2014;21(4):e63-6.

13. Emiralioglu N, Dogru D, Yalcin S, Tugcu GD, Yalcin E, Ozcelik U, Ekinci S, Kiper N. Impact of surgery on growth, pulmonary functions, and acute pulmonary exacerbations in children with non-cystic fibrosis bronchiectasis. Thorac Cardiovasc Surg. 2019;67(01):058-66. 
14. Lucas JS, Burgess A, Mitchison HM, Moya E, Williamson M, Hogg C, National PCD, Service U. Diagnosis and management of primary ciliary dyskinesia. Arch Dis Child. 2014;99(9):850-6.

15. Smit HJ, Schreurs AJ, Van den Bosch JMM, Westermann CJ. is resection of bronchiectasis beneficial in patients with primary ciliary dyskinesia? Chest. 1996;109(6):1541-4.

16. Goutaki M, Maurer E, Halbeisen FS, Amirav I, Barbato A, Behan L, Boon M, Casaulta C, Clement A, Crowley S, Haarman E, Hogg C, Karadag B, KoernerRettberg C, Leigh MW, Loebinger MR, Mazurek H, Morgan L, Nielsen KG, Omran H, Schwerk N, Scigliano S, Werner C, Yiallouros P, Zivkovic Z, Lucas JS, Kuehni CE, PCD Italian Consortium, Swiss PCD Group, French Reference Centre for Rare Lung Diseases, Genetic Disorders of Mucociliary Clearance Consortium. The international primary ciliary dyskinesia cohort (iPCD Cohort): methods and first results. Eur Respir J. 2017:49(1). https://doi.org/10. 1183/13993003.01181-2016 Print 2017 Jan.

17. Gail MH. Frequency matching. Statistics Reference Online: Wiley StatsRef; 2005.

18. Werner C, Onnebrink JG, Omran H. Diagnosis and management of primary ciliary dyskinesia. Cilia. 2015;4(1):2.

19. Lucas JS, Barbato A, Collins SA, Goutaki M, Behan L, Caudri D, Dell S, Eber E, Escudier E, Hirst AR, Hogg C, Jorissen M, Latzin P, Legendre M, Leigh MW, Midulla F, Nielsen KG, Omran H, Papon JF, Pohunek P, Redfern B, Rigau D, Rindlisbacher B, Santamaria F, Shoemark A, Snijders D, Tonia T, Titieni A, Walker WT, Werner C, Bush A, Kuehni CE. European Respiratory Society guidelines for the diagnosis of primary ciliary dyskinesia. Eur Respir J. 2017; 49(1):1601090.

20. Becker SO, Ichino A. Estimation of average treatment effects based on propensity scores. Stata J. 2002;2(4):358-77.

21. Jain K, Padley S, Goldstraw E, Kidd S, Hogg C, Biggart E, Bush A. Primary ciliary dyskinesia in the paediatric population: range and severity of radiological findings in a cohort of patients receiving tertiary care. Clin Radiol. 2007;62(10):986-93.

22. Cohen-Cymberknoh M, Simanovsky N, Hiller N, Hillel AG, Shoseyov D, Kerem E. Differences in disease expression between primary ciliary dyskinesia and cystic fibrosis with and without pancreatic insufficiency. Chest J. 2014;145(4):738-44.

23. Maglione M, Montella S, Mollica C, Carnovale V, lacotucci P, De Gregorio F, Tosco A, Cervasio M, Raia V, Santamaria F. Lung structure and function similarities between primary ciliary dyskinesia and mild cystic fibrosis: a pilot study. Ital J Pediatr. 2017;43(1):34

24. Dettmer S, Ringshausen F, Vogel-Claussen J, Fuge J, Faschkami A, Shin H, Schwerk N, Welte T, Wacker F, Rademacher J. Computed tomography in adult patients with primary ciliary dyskinesia: typical imaging findings. PLoS One. 2018;13(2):e0191457.

25. Tadd K, Morgan L, Rosenow T, Schultz A, Susanto C, Murray C, Robinson P. CF derived scoring systems do not fully describe the range of structural changes seen on CT scans in PCD. Pediatr Pulmonol. 2019. https://doi.org/ 10.1002/ppul.24249.

26. Priftis KN, Mermiri D, Papadopoulou A, Anthracopoulos MB, Vaos G, Nicolaidou P. The role of timely intervention in middle lobe syndrome in children. Chest. 2005;128(4):2504-10.

27. Einarsson JT, Einarsson JG, Isaksson H, Gudbjartsson T, Gudmundsson G. Middle lobe syndrome: a nationwide study on clinicopathological features and surgical treatment. Clin Respir J. 2009;3(2):77-81.

28. Frija-Masson J, Bassinet L, Honore I, Dufeu N, Housset B, Coste A, Papon JF, Escudier E, Burgel PR, Maitre B. Clinical characteristics, functional respiratory decline and follow-up in adult patients with primary ciliary dyskinesia. Thorax. 2017;72(2):154-60.

29. Shah A, Shoemark A, MacNeill SJ, Bhaludin B, Rogers A, Bilton D, Hansell DM, Wilson R, Loebinger MR. A longitudinal study characterising a large adult primary ciliary dyskinesia population. Eur Respir J. 2016;48(2):441-50.

30. Goutaki M, Halbeisen FS, Spycher BD, Maurer E, Belle F, Amirav I, Behan L, Boon M, Carr S, Casaulta C, Clement A, Crowley S, Dell S, Ferkol T, Haarman EG, Karadag B, Knowles M, Koerner-Rettberg C, Leigh MW, Loebinger MR, Mazurek H, Morgan L, Nielsen KG, Phillipsen M, Sagel SD, Santamaria F, Schwerk N, Yiallouros P, Lucas JS, Kuehni CE, PCD Israeli Consortium, Swiss PCD Group, French Reference Centre for Rare Lung Diseases. Growth and nutritional status, and their association with lung function: a study from the international Primary Ciliary Dyskinesia Cohort. Eur Respir J. 2017;50(6). https://doi.org/10.1183/13993003.01659-2017 Print 2017 Dec.

31. Aurora P, Wade A, Whitmore P, Whitehead B. A model for predicting life expectancy of children with cystic fibrosis. Eur Respir J. 2000;16(6):1056-60.
32. McColley SA, Schechter MS, Morgan WJ, Pasta DJ, Craib ML, Konstan MW. Risk factors for mortality before age 18 years in cystic fibrosis. Pediatr Pulmonol. 2017:52(7):909-15.

33. Miller MR, Hankinson JATS, Brusasco V, Burgos F, Casaburi R, Coates A Crapo $R$, Enright $P$, van der Grinten CPM, Gustafsson P, Jensen $R$, Johnson DC, Maclntyre N, McKay R, Navajas D, Pedersen OF, Pellegrino R, Viegi G, Werner J. Standardisation of spirometry. Eur Respir J. 2005;26(2):319-38.

\section{Publisher's Note}

Springer Nature remains neutral with regard to jurisdictional claims in published maps and institutional affiliations.
Ready to submit your research? Choose BMC and benefit from:

- fast, convenient online submission

- thorough peer review by experienced researchers in your field

- rapid publication on acceptance

- support for research data, including large and complex data types

- gold Open Access which fosters wider collaboration and increased citations

- maximum visibility for your research: over $100 \mathrm{M}$ website views per year

At BMC, research is always in progress.

Learn more biomedcentral.com/submissions 